\title{
Synthesis of One Dimensional Gold Nanostructures
}

\author{
Hongchen Li, ${ }^{1}$ Caixia Kan, ${ }^{1}$ Zhaoguang Yi, ${ }^{1}$ Xiaolong Ding, ${ }^{1}$ \\ Yanli Cao, ${ }^{1}$ and Jiejun $\mathrm{Zhu}^{2}$ \\ ${ }^{1}$ College of Science, Nanjing University of Aeronautics and Astronautics, Nanjing 211100, China \\ ${ }^{2}$ National Laboratory of Solid State Microstructures and Department of Physics, Nanjing University, Nanjing 210093, China
}

Correspondence should be addressed to Caixia Kan, cxkan@nuaa.edu.cn

Received 25 September 2010; Accepted 2 December 2010

Academic Editor: Sherine Obare

Copyright () 2010 Hongchen Li et al. This is an open access article distributed under the Creative Commons Attribution License, which permits unrestricted use, distribution, and reproduction in any medium, provided the original work is properly cited.

\begin{abstract}
Gold nanostructures with shapes of rod, dumbbells, and dog bone have been fabricated by an improved seed-mediated method. It is found that the $\mathrm{pH}$ change (the addition of $\mathrm{HNO}_{3}$ or $\mathrm{HCl}$ ) and the presence of $\mathrm{Ag}^{+}$ions have a great influence on the growth process and aspect ratios of these Au nanocrystals. UV-Vis-NIR absorption spectra for the Au colloidal show that the transverse plasmon absorption band locates at $\sim 520 \mathrm{~nm}$, while the longitudinal plasmon absorption band shifts in a wide spectra region of 750-1100 nm. The obtained Au nanostructures have been investigated by transmission electron microscopy, high-resolution transmission electron microscopy, and X-ray diffractometer. Based on the characterizations and FDTD simulations, most of the obtained Au nanorods are single crystals, possessing an octagonal cross-section bounded by $\{110\}$ and $\{100\}$ faces. One model for the anisotropic growth has been proposed. It is found that slow kinetics favor the formation of single-crystalline Au nanorods.
\end{abstract}

\section{Introduction}

Noble metallic nanostructures are of great interest due to their unique properties and promising applications in the fields of optics, electronics, magnetism, and catalysis [16]. Pt nanoparticles, for instance, were applied as catalyst for the hydrogenation of organic and for selective reactions depending on the crystallographic planes exposed by their surfaces $[5,6]$. For the case of optical properties, absorption associated with the collective oscillation of the conduction electrons or surface plasmon resonance (SPR) has been studied for many decades. In recent years, several special shapes and structures have been studied for $\mathrm{Au}$ nanoparticles, such as nanorings, nanoplates, dog bones, and nanoprisms. At present, the SPR absorption for arbitrary geometries can be theoretically calculated by discrete dipole approximation or finite difference time domain (FDTD) solutions (FDTD supplies a simple, convenient, and systematic approach to calculate the optical response of a nanostructure with arbitrary symmetry and geometry by solving Maxwell's equations on discrete grids). Both experimental and theoretical studies show that the number and position of the plasmon resonances as well as the spectral range for surface-enhanced Raman scattering (SERS) of metal nanostructures have a strong correlation with their exact morphology and the aspect ratios [1, 7-12]. For example, cylindrical Ag nanowires show one resonance whereas several resonances are expected for Ag nanorods with triangle and other profiles [8,9]. Two distinctive plasma resonances usually appear in the optical absorption spectra. In addition to a weak transverse surface plasmon resonance $\left(\mathrm{SPR}_{\mathrm{T}}\right)$ roughly in the visible spectral region (500$530 \mathrm{~nm}$ with different solvents), a strong longitudinal surface plasmon resonance $\left(\mathrm{SPR}_{\mathrm{L}}\right)$ shifts from the visible to the nearinfrared (Vis-NIR) region with increasing aspect ratios of Au nanorods [10-12]. This strong polarization sensitivity of SPR absorption in the NIR region is an efficient converter of photon energy to thermal energy, which opens new possibilities for many attractive applied fields. Due to their facile synthesis, ease of functionalization, biocompatibility, and inherent nontoxicity, Au nanoparticles are being developed as ideal biological applications, such as gene delivery, cell imaging, photothermal therapy, and anticancer drugdelivery technology [1,13-17]. More recently, it is reported five-dimensional (the wavelength, polarization, and 3 spatial dimensions) optical recording mediated by the SPR of Au 
nanorods, which allow multiple patterns being stored in the same volume [18]. Under irradiating recording layers doped with $\mathrm{Au}$ nanorods of different aspect ratios, the selected nanorods with a certain $\mathrm{SPR}_{\mathrm{L}}$ on resonance with the laser light wavelength and polarization would melt. When the readout laser light is implemented, the contrast exhibits.

For fundamental and applied interest, study on the wellcontrolled shapes and novel structures of Au nanostructures, therefore, has become a very important issue. For one dimensional Au nanorods, different approaches have been demonstrated for synthesizing Au nanorods, including hard templates (such as $\mathrm{Al}_{2} \mathrm{O}_{3}$ membrane and mesoporous silica) assistant deposition approach $[12,19]$, electrochemical or photochemical reduction with surfactants [20,21], and seedmediated methods [22]. Recently, some groups reported the synthesis of branched and multipod-shaped metal nanostructures with sharp edges and corners [23-27]. Calculation on the fields around nanoparticles show that surface charges are accumulated at the sharp corners that exhibit strong enhancement of an electromagnetic field [28], which makes the nanostructure an excellent candidate as SERS substrates. At the same time, contrast studies on the SPR of these quasione-dimensional Au nanostructures demonstrate that the shape and position of the $\mathrm{SPR}_{\mathrm{L}}$ was not only sensitive to the aspect ratio but also influenced by additional details of the rod shape and in particular by the natural transition from cylindrical to flared, dog bone, bipyramidal, and dumbbell [29-32].

Here, we report the synthesis of Au nanorods, dumbbells, and dog bone through an improved seed-mediated at room temperature. It is found that the addition of acid, $\mathrm{Ag}^{+}$ ions and a second surfactant is crucial in both improving the shape and controlling the aspect radios of $\mathrm{Au}$ product. A growth model was proposed based on the results.

\section{Experimental Section}

2.1. Materials. Hydrogen tetrachloroaurate $\left(\mathrm{HAuCl}_{4} \cdot 4 \mathrm{H}_{2} \mathrm{O}\right)$ was purchased from Shanghai Chemical Reagents Company. L-ascorbic acid (AA, 99.7\%) and sodium borohydride $\left(\mathrm{NaBH}_{4}, 96 \%\right)$ were obtained from Sinopharm Chemical Reagent Company. Cationic cetyltrimethylammonium bromide (CTAB, 99\%) and benzyldimethylhexadecyl ammonium chloride (BDAC) were obtained from Nanjing Robiot Company. All other reagents were used without further purification. Deionized water used throughout the experiments was purified by a MilliQ system (18.25 M $\Omega$ ).

2.2. Synthesis. Seed solutions were generated firstly with a strong reducing agent $\left(\mathrm{NaBH}_{4}\right)$, followed by preparation of a growth solution with a weaker reducing agent (AA) to grow $\mathrm{Au}$ nanorods. In the improved process, chemicals of $\mathrm{HNO}_{3}$, $\mathrm{AgNO}_{3}$, and BDAC were applied in the growth solution for the understanding of growth mechanism of final product. The details of the process are the following.

2.2.1. Synthesis of $A u$ Seeds. $10 \mathrm{~mL}$ CTAB (with molar concentration $[\mathrm{CTAB}]=0.1 \mathrm{M}$ ) was mixed with $0.05 \mathrm{~mL} \mathrm{HAuCl}_{4}$ solution $\left(\left[\mathrm{HAuCl}_{4}\right]=0.05 \mathrm{M}\right)$. Then, $0.6 \mathrm{~mL} \mathrm{NaBH} 4$ $\left(\left[\mathrm{NaBH}_{4}\right]=0.01 \mathrm{M}\right)$ was injected into the solution under vigorous stirring. The as-obtained seeds are very active indicated by the color change from brownish to red within one day.

2.2.2. Synthesis of Au Dog Bones, Dumbbells, and Nanorods. CTAB was dissolved into $10 \mathrm{~mL}$ deionized water $([\mathrm{CTAB}]=$ $0.1 \mathrm{M})$ as growth solution. Then, solutions of $0.2 \mathrm{~mL} \mathrm{AgNO}_{3}$ $\left(\left[\mathrm{AgNO}_{3}\right]=0.004 \mathrm{M}\right)$ and $0.1 \mathrm{~mL} \mathrm{HAuCl} \mathrm{H}_{4}\left(\left[\mathrm{HAuCl}_{4}\right]=\right.$ $0.05 \mathrm{M}$ ) were added to the growth solution. When $0.06 \mathrm{~mL}$ $\mathrm{AA}([\mathrm{AA}]=0.1 \mathrm{M})$ was added, the solution color changed from orange to colorless. Finally, $12 \mu \mathrm{L}$ of the as-prepared seed solution was injected into the growth solution. Under this condition, Au nanostructures with shapes of dog bones and dumbbells were synthesized. In order to fabricate $\mathrm{Au}$ nanorods, different amount $(0.1 \mathrm{~mL}, 0.2 \mathrm{~mL}, 0.3 \mathrm{~mL}$, and $0.4 \mathrm{~mL})$ of acid $\left(\mathrm{HNO}_{3}\right.$ or $\mathrm{HCl}$ with concentration of $\left.1 \mathrm{M}\right)$ was employed in the growth solution. When BDAC was used as a cosurfactant, Au nanorods with large aspect ratios were obtained.

2.3. Characterization. The absorption spectra of the prepared samples were collected using a UV-Vis-NIR spectrometer (SP-752PC) in the wavelength range of 200-1100 nm. The products were purified by centrifugation at $14000 \mathrm{rpm}$ for $20 \mathrm{~min}$. Precipitates were centrifugated repeatedly with deionized water. Then, the samples were deposited on copper grids covered by an amorphous carbon film, HRTEM grids, and glass slides for further measurements. Microscopic observations were carried out using transmission electron microscope (TEM: JEOL-100CX) and high-resolution TEM (HRTEM: JEOL-2011). XRD measurement was performed on a diffractometer (Ultima-III, Rigaku).

\section{Results and Discussion}

3.1. Microstructure and Optical Absorption of Au Nanostructures. As described in the experimental section, Au dog bones and nanorods were prepared, respectively, in the absence and with the presence of $\mathrm{HNO}_{3}$ with $\mathrm{CTAB}$ as surfactant. Figure 1(a) shows the UV-Vis-NIR absorption spectra for the $\mathrm{Au}$ colloids of dog bones and nanorods. In contrast to $\mathrm{Au}$ nanorods, the $\mathrm{SPR}_{\mathrm{T}}$ of dog-bones is wide. Upon addition of $\mathrm{HNO}_{3}$, the $\mathrm{SPR}_{\mathrm{L}}$ position changes from $767 \mathrm{~nm}$ to $860 \mathrm{~nm}$, indicating the aspect ratios of $\mathrm{Au}$ nanorods increase. It is found that the reduction rate of $\mathrm{Au}^{3+}$ ions decreases when acid $\left(\mathrm{HNO}_{3}\right.$ or $\left.\mathrm{HCl}\right)$ is added in the growth solution, which facilitates the formation of $\mathrm{Au}$ nanorods with large aspect ratios. The structures of the products were characterized by TEM measurements. The SAED patterns indicate the single-crystalline nature of the obtained $\mathrm{Au}$ nanocrystals. The dog bone is measured to be $50 \mathrm{~nm}$ in average length and $11 \mathrm{~nm}$ in diameter for the middle section, and some of the products take the shapes of dumbbell and cube, as exhibited in Figure 1(b). The aspect ratio for $\mathrm{Au}$ nanorods in Figure 1 (c) is $\sim 4.5$. Usually, Au nanorods were available over Au dog bones by 


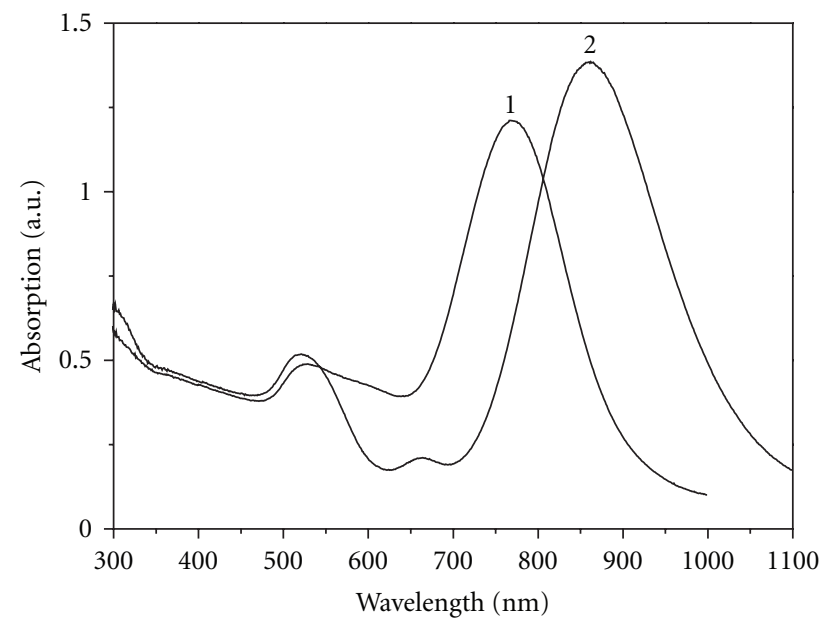

(a)

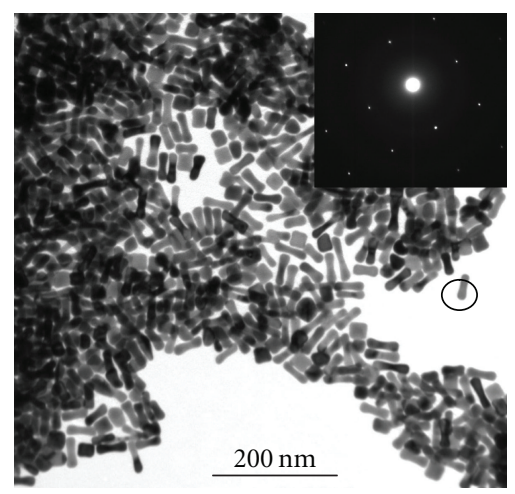

(b)

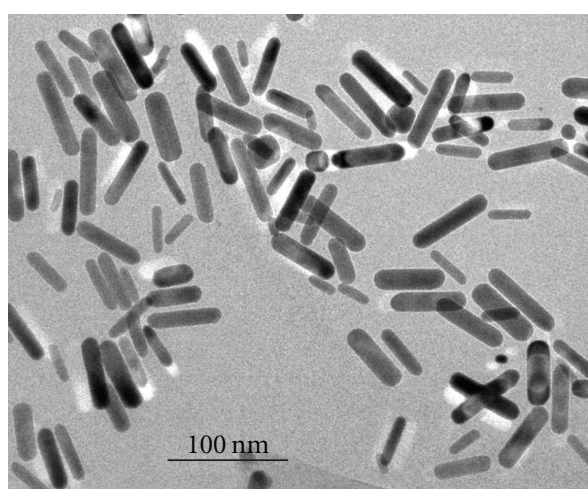

(c)

FIgURE 1: (a) Vis-NIR absorption spectra of Au dog bones (curve-1) and Au nanorods (curve-2) prepared in the absence and with the presence of $\mathrm{HNO}_{3}$, respectively. (b) and (c) TEM images and SAED pattern of Au dog bones and Au nanorods corresponding to spectra 1 and spectra 2 , respectively.

decreasing the content of $\mathrm{AA}$ or increasing the content of "seed" solution even in the absence of acid [33, 34]. Instead of these two strategies, it confirms that the addition of $\mathrm{HNO}_{3}$ is more efficient in modifying the dog bone in our current work. Additionally, the chemical CTAB purchased from different supplies would lead to quite different results; sometimes no Au nanorods were obtained [35]. This could be attributed to the difference of capping ability which significantly influences the morphology of the final products.

To produce $\mathrm{Au}$ nanorods with large aspect ratios, a surfactant mixture contained $\mathrm{CTAB}$ and BDAC ([BDAC]/ $[\mathrm{CTAB}]=1.25)$ was applied in the growth solution. Figure 2(a) shows the UV-Vis-NIR absorption spectra for the colloid of $\mathrm{Au}$ nanorods produced with the presence of $\mathrm{CTAB} / \mathrm{BDAC}$ sampled at different stages. It is clear that the absorption intensity of Au nanostructure increases during aging, suggesting the increase of number and (or) volume of $\mathrm{Au}$ nanostructures. The red shift of the $\mathrm{SPR}_{\mathrm{L}}$ position indicates that the aspect ratio of $\mathrm{Au}$ nanorods increases with time, which is opposite to the reported trend [36]. The recorded spectra also show that Au nanorods maintain their overall shapes during growth process, implying proper rate of Au supply in the colloidal solution. Thus, proper reduction rate favors the formation of nanorods. For the growth of $\mathrm{Au}$ dog bones and dumbbells, the formation originates additional deposition of Au cluster at the ends of the as-formed nanorods which could act as seeds.

In our experiments, the presence of $\mathrm{HNO}_{3}$ or $\mathrm{HCl}$ plays an important role in synthesizing Au nanorods. For fully understanding the role of acid, a series of syntheses were performed by altering the content of $\mathrm{HNO}_{3}$. As shown in Figure 2(b) for samples after addition of seed solution for $\sim 24 \mathrm{~h}$, the SPR $\mathrm{L}$ band blue shifts with increase the content of $\mathrm{HNO}_{3}$ from 0.1 to $0.4 \mathrm{~mL}$, which offers a useful means to systematically control the shape and aspect ratios of $\mathrm{Au}$ nanostructures. Further results show that $\mathrm{HNO}_{3}$ can slow down the growth rate effectively. For example, the solution became light red after $3 \mathrm{~h}$ when $0.2 \mathrm{~mL} \mathrm{HNO}_{3}$ was added, while $5 \mathrm{~h}$ was needed for this change with addition of $0.3 \mathrm{~mL} \mathrm{HNO}_{3}$. When $\mathrm{HCl}$ was applied, similar results were observed. In the TEM image of Au nanorods, several Au nanocubes were also observed, together with some small nanoparticles as byproducts, as shown in Figure 2(c). The aspect ratio of these nanorods is estimated to be 


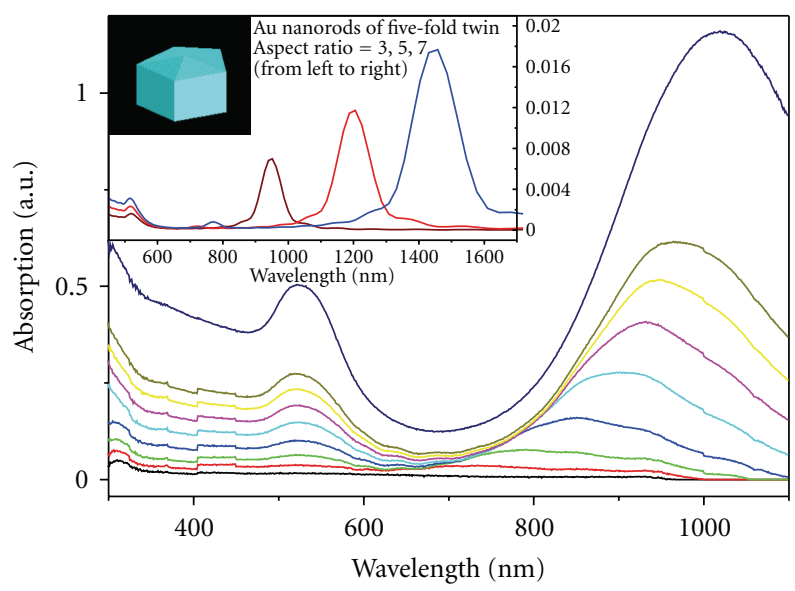

(a)

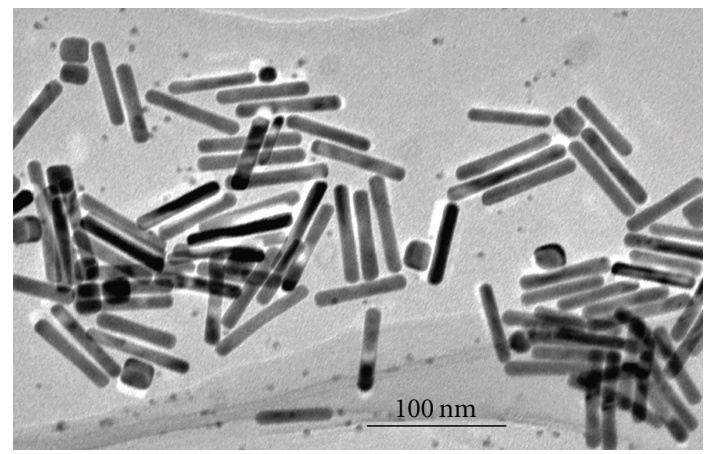

(c)

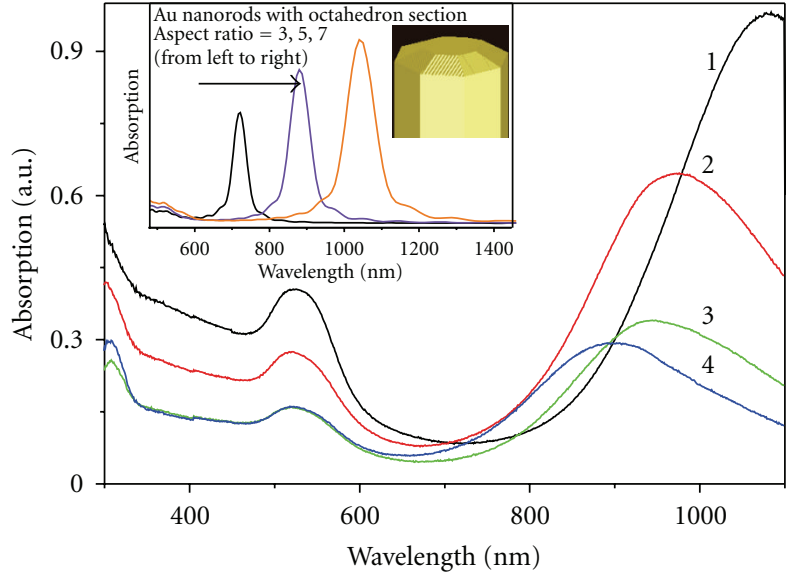

(b)

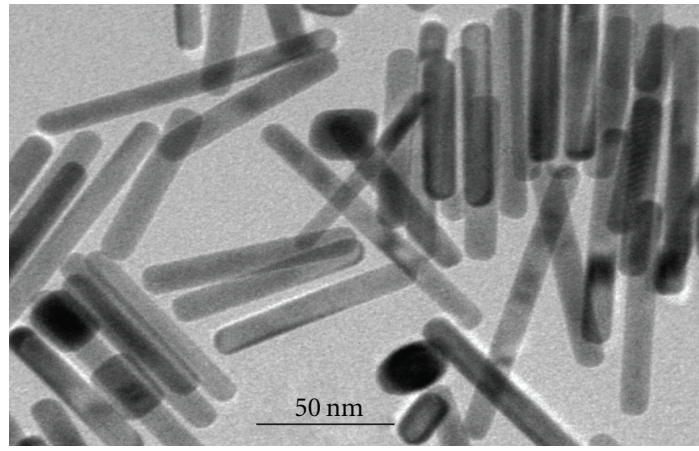

(d)

Figure 2: (a) Vis-NIR spectra of colloidal Au nanorods aged for various times. Spectra from bottom to top correspond to samples aged for 1-8 hour and 1 day. (b) Vis-NIR spectra of 4 identical growth solutions with increasing $\mathrm{HNO}_{3}$ (curve-1: $0.1 \mathrm{~mL}$, curve-2: $0.2 \mathrm{~mL}$, curve-3: $0.3 \mathrm{~mL}$, and curve-4: $0.4 \mathrm{~mL}$ ). Insets of (a) and (b) are the calculated spectra using FDTD solution for Au nanorods with fivefold twin and octahedron cross section (water as medium). TEM images of Au nanorods prepared with $\mathrm{HNO}_{3}(0.1 \mathrm{~mL})(\mathrm{c})$ and $\mathrm{HCl}(0.2 \mathrm{~mL})(\mathrm{d})$ in a binary surfactants of CTAB and BDAC.

$\sim 7$ (the average lengths and diameters of nanorods are, resp., $\sim 70 \mathrm{~nm}$ and $\sim 10 \mathrm{~nm}$ for Figure 2(c) and $\sim 84 \mathrm{~nm}$ and $\sim 12 \mathrm{~nm}$ for Figure $2(\mathrm{~d})$ ), corresponding to the $\mathrm{SPR}_{\mathrm{L}}$ band position at $1050 \mathrm{~nm}$. Inserted in Figures 2(a) and 2(b) are the FDTD calculated optical absorption spectra for the fivefold twined $\mathrm{Au}$ nanorods and $\mathrm{Au}$ nanorods with octahedron cross section (with aspect ratios of 3,5, and 7, using water as medium). The simulations indicate that the obtained $\mathrm{Au}$ nanorods are not five-fold twined crystal, but singlecrystalline Au nanorods with octahedron cross section.

Figure 3 shows HRTEM images of representative Au nanorods and dumbbells. The SAED patterns indicate that these structures are single crystals. Au nanorods appear to grow along [001] direction with side faces bounded by $\{100\}$ and $\{110\}$ facets. Clear fringes parallel and perpendicular to the growth axis are shown in Figure 3(a). The fringe spacing is $0.200 \mathrm{~nm}$, corresponding well with the lattice spacing of $\{200\}$ planes for Au crystal $(0.203 \mathrm{~nm})$. Similar structures are found for Au dumbbells. Occasionally, the presence of $\{111\}$ facets were observed at two ends of Au dumbbell, as detailed in Figure 3(b).

To further determine the structure characterization of the $\mathrm{Au}$ nanorods, an X-ray diffraction experiment was carried out. Figure 4 shows the typical XRD result of one sample. In the XRD profile, four diffraction peaks can be indexed to fcc $\mathrm{Au}$ crystal though the background information is clear due to a very thin layer of $\mathrm{Au}$ nanorods covered on the quartz glass substrate. The XRD profile of the Au nanorods shows a strong (111) diffraction peak, and the intensity of the (200) diffraction peak is also strong. The enhanced (111) and (200) diffractions indicate that the stack and elongation of $\{111\}$ and $\{100\}$ facets mainly account for the crystals growth.

\subsection{Growths of One Dimensional Au Nanocrystals}

3.2.1. The Role of Halogen Ions. According to [37], ion addictives, such as $\mathrm{NaCl}, \mathrm{KCl}$, and $\mathrm{NaNO}_{3}$, can serve as useful "tools" to tailor the shape, aspect ratio, and yield of $\mathrm{Au}$ nanorods. These addictives caused the $\mathrm{SPR}_{\mathrm{L}}$ band red shift within the critical concentrations. Ionic strength in colloidal solution has an effect on the size of soft template and suitable ionic concentration can improve micellar structure through reducing the repulsion between the neighboring head group of CTAB [38]. However, further increasing the ionic concentration may prompt a template transfiguration from rod to bone. A series of studies, concentrated on 


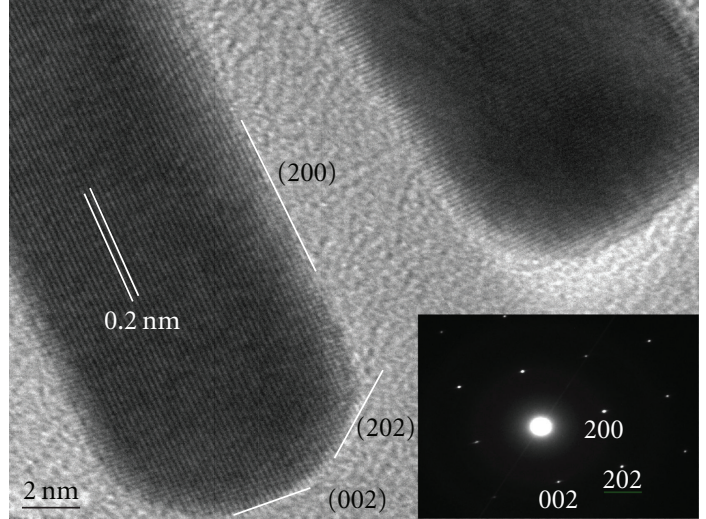

(a)

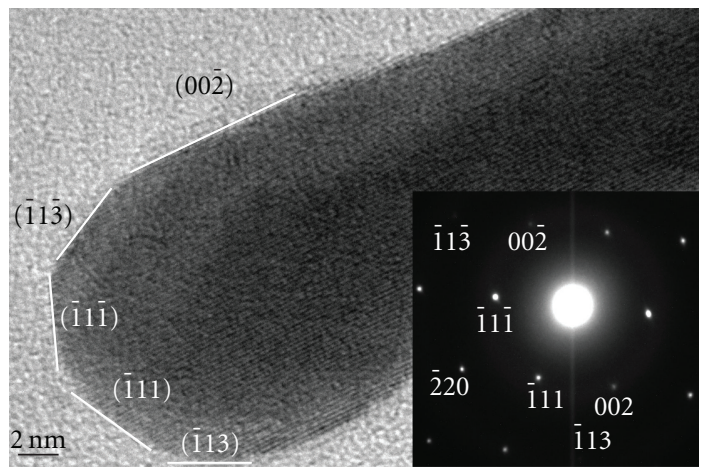

(b)

FIGURE 3: (a) HRTEM image of two Au nanorods recorded along [100] direction. The inset shows the corresponding SAED pattern. (b) HRTEM image of one Au dumbbell recorded along [110] direction. The inset shows the corresponding SAED pattern.

$\mathrm{Br}^{-}$ions pointed out that $\mathrm{Br}^{-}$ions aided the formation of structures covered with $\{110\}$ and $\{100\}$ facets $[39,40]$. We suggest that the major function of halogen is chemisorption on Au crystal surface or chemical interaction between $\mathrm{Au}$ crystals and surfactant. Proper concentration of halogen ions avails the formation of single-crystalline Au nanorods with large aspect ratios.

3.2.2. The Role of $\mathrm{Ag}^{+}$Ions. Originally, the seed-mediated method without addition of $\mathrm{AgNO}_{3}$ was reported by Jana et al. Au nanorods with high aspect ratio $(\sim 18)$ were obtained for citrate-capped seeds, but the yield was very low [41]. Then, this method was improved to increase the yield of $\mathrm{Au}$ nanorods through adding $\mathrm{Ag}^{+}$ions to the growth solution [42]. In addition to a high yield of Au nanorods, the $\mathrm{SPR}_{\mathrm{L}}$ can be tuned in a wide wavelength range upon increasing the concentration of $\mathrm{Ag}^{+}$ions. Based on the soft template of $\mathrm{CTAB}, \mathrm{AgBr}$ will be formed with addition of $\mathrm{AgNO}_{3}$, which would decrease the charge density of adsorbed CTAB molecules on Au facets and the consequent repulsion between the neighboring head groups, favoring the CTAB template elongation. Another model is that $\mathrm{AgBr}$ would adsorb preferentially on special facets and direct the rod-shape growth $[33,39]$. Therefore, ideas can be expected

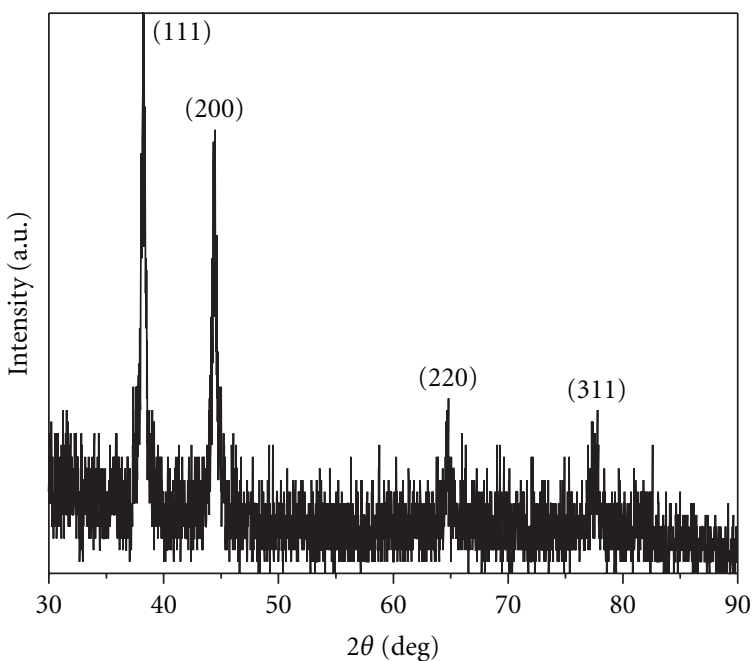

FIGURE 4: X-ray diffraction profile of the as-prepared Au nanorods.

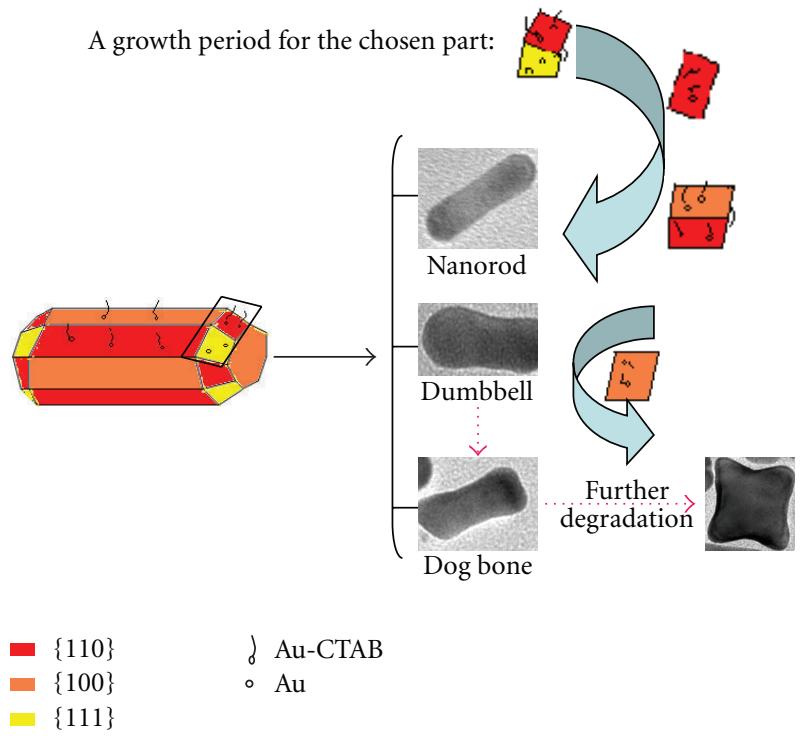

FIGURE 5: Schematic illustration of proposed growth mechanism responsible for the formation of nanorods, dumbbells, and dog bones.

for the application of $\mathrm{AgNO}_{3}$. (i) $\mathrm{AgBr}$ adsorbs on the single crystalline Au "seed", promoting anisotropic growth into nanorods. (ii) $\mathrm{AgNO}_{3}$ has an effect on making Au nanorods stable and protecting them from evolving into other shapes. Otherwise, when no $\mathrm{AgNO}_{3}$ was introduced, the growth solution turned red within 1 min after the addition of "seed" solution, and the product is dominated by Au nanospheres. In the experiment results, no $\mathrm{Ag}$ nanostructures were obtained. This should ascribe to the fact that $\mathrm{Ag}^{+}$cannot be reduced in the slightly acidic growth conditions (due to the fact that any $\mathrm{AuCl}_{4}{ }^{-}$present is an oxidant with respect to $\operatorname{Ag}(0))$.

3.2.3. The Role of $C T A B$ and BDAC. Undoubtedly, the key factor for prompting the formation of $\mathrm{Au}$ nanorods is the 
molecules of CTAB. CTAB possesses an ammonium headgroup and a hydrocarbon tail which maybe a driven force for the combination between $\mathrm{Au}$ and $\mathrm{CTA}^{+}$. CTAB forms micelles above its critical micelle concentration. It is widely accepted that CTAB prefer to bind to the side surface of $\mathrm{Au}$ nanorods through $\mathrm{Br}^{-}$ions chemisorbing on the side surfaces [43]. Thus, the side surface is well protected from further deposition. When all conditions remained the same except for the length of the surfactant tail, Au nanorods with high aspect ratios were obtained upon increasing the length of surfactant chain $[39,44]$. Others proposed that $\mathrm{CTAB}$ micelles acted as a soft template for controlling the shape and size of particles. Au atoms reduced were trapped by the preformed templates, leading to the formation of $\mathrm{Au}$ nanorods $[37,38]$. In our experiment, solutions of $C T A B$ and $\mathrm{CTAB} / \mathrm{BDAC}$ were prepared and kept at $10^{\circ} \mathrm{C}$. $\mathrm{CTAB}$ precipitate was observed in the solution within $30 \mathrm{~min}$, but it took much longer time for the appearance of CTAB precipitate in the mixture solution. This observation demonstrated that the solubility of $\mathrm{CTAB}$ is improved dramatically in the $\mathrm{CTAB} / \mathrm{BDAC}$ mixture solution, and that the templates became more flexible, as evidenced by the elongation of $\mathrm{Au}$ nanorods upon aging. Moreover, it is found that the growth of Au nanorods is more time saving in the single-component surfactant than in the binary surfactant mixture.

3.2.4. Growth Model for Different Shapes. For the case of crystal growth in solution phase, the final structure of crystal is determined by both kinetic and thermodynamic effect $[9,45-47]$. It is known that thermodynamic control always attempts to minimize the total interfacial free energy of a crystal. However, in the chemical synthesis with the presence of surfactant, the slow reduction rate of $\mathrm{Au}^{3+}$ ions provides the possibility for the highly anisotropic growth. The coverage of $\{110\}$ facets on nanorods seems to deviate from thermodynamic control. This should ascribe to that the surfactant CTAB molecular usually binds to $\{110\}$ facet with preference, and that the Au atom spacing on the side faces $(\{110\}$ or $\{100\})$ is comparable to the size of the $\mathrm{CTA}^{+}$headgroup $[39,48]$. Therefore, the single-crystalline Au nanorods have an octagonal cross-section covered by flat $\{100\}$ facets (the truncated corners are bounded by $\{111\}$ and $\{110\}$ facets) with sidewalls of $\{110\}$ and $\{100\}$ facets.

In our postulation, the free $\mathrm{Au}$ and $\mathrm{Au}-\mathrm{CTAB}$ complex coexist in the growth solution with different affinity to crystal facets. The Au-CTAB complex are preferred to the $\{110\}$ facets at the corner of Au rods, while the free Au to the $\{111\}$ facets. The deposition rate of free $\mathrm{Au}$ on $\{111\}$ facets is faster than that of the bonded $\mathrm{Au}$ on $\{110\}$ facets, which results in the elongation of $\{110\}$ facets. The growth mechanism of these Au nanostructures can be depicted as schematically illustrated in Figure 5. The $\{110\}$ facets are not thermodynamically stable and easily evolve into $\{100\}$ facets with the aid of surfactants, forming the octagonal cross-section covered by both $\{110\}$ and $\{100\}$ facets. With increasing the kinetic rate, the rods change from a cylindrical form to a dumbbell and further to dog-bone. A large kinetic rate facilitates the appearance of $\{100\}$ facets and thus, dog bones dominate the products and then degrade into the cubes.

\section{Conclusions}

In summary, we conducted the synthesis of single-crystalline Au nanorods, dumbbells, and dog bones in solution phase that contains surfactants with the addition of acid. Through comparing the results, we can see that changes of $\mathrm{PH}$ value (by adding $\mathrm{HNO}_{3}$ and $\mathrm{HCl}$ ) can modify the morphology and tune the aspect ratios of the final products. The $S P R_{L}$ of Au nanorods can be tuned in a large spectra range of 750$1100 \mathrm{~nm}$, which is important for the potential applications related to this property. A growth model is proposed, in which kinetics and thermodynamics are employed.

\section{Acknowledgments}

This work was financially supported from National Natural Science Foundation of China (Grant nos. 10704038, 10772084, and 51032002). Authors thank Dr. Kai Shen of College of Materials Science of NUAA and Dr. Xiaoguang Zhu of Institute of Solid State Physics of CAS for their great help on HRTEM measurement.

\section{References}

[1] K. L. Kelly, E. Coronado, L. L. Zhao, and G. C. Schatz, "The optical properties of metal nanoparticles: the influence of size, shape, and dielectric environment," Journal of Physical Chemistry B, vol. 107, no. 3, pp. 668-677, 2003.

[2] B. J. Wiley, Z. Wang, J. Wei, Y. Yin, D. H. Cobden, and Y. Xia, "Synthesis and electrical characterization of silver nanobeams," Nano Letters, vol. 6, no. 10, pp. 2273-2278, 2006.

[3] C. B. Murray, S. Sun, H. Doyle, and T. Betley, "Monodisperse 3d Transition-metal (Co, Ni, Fe) nanoparticles and their assembly into nanoparticle superlattices," MRS Bulletin, vol. 26, no. 12, pp. 985-991, 2001.

[4] G. A. Somorjai and D. W. Blakely, "Mechanism of catalysis of hydrocarbon reactions by platinum surfaces," Nature, vol. 258, no. 5536, pp. 580-583, 1975.

[5] A.-C. Shi and R. I. Masel, "The effects of gas adsorption on particle shapes in supported platinum catalysts," Journal of Catalysis, vol. 120, no. 2, pp. 421-431, 1989.

[6] H. Wu, R. Tang, Q. He, X. Liao, and B. Shi, "Highly stable Pt nanoparticle catalyst supported by polyphenolgrafted collagen fiber and its catalytic application in the hydrogenation of olefins," Journal of Chemical Technology and Biotechnology, vol. 84, no. 11, pp. 1702-1711, 2009.

[7] J. M. McLellan, Z. Y. Li, A. R. Siekkinen, and Y. Xia, "The SERS activity of a supported ag nanocube strongly depends on its orientation relative to laser polarization," Nano Letters, vol. 7, no. 4, pp. 1013-1017, 2007.

[8] P. J. Kottman, O. J. F. Martin, D. R. Smith, and S. Schultz, "Plasmon resonances of silver nanowires with a nonregular cross section," Physical Review B, vol. 64, no. 23, Article ID 235402, 10 pages, 2001.

[9] C.-X. Kan, J.-J. Zhu, and X.-G. Zhu, "Silver nanostructures with well-controlled shapes: synthesis, characterization and growth mechanisms," Journal of Physics D, vol. 41, no. 15, Article ID 155304, 2008. 
[10] J. H. Song, F. Kim, D. Kim, and P. Yang, "Crystal overgrowth on gold nanorods: tuning the shape, facet, aspect ratio, and composition of the nanorods," Chemistry: A European Journal, vol. 11, no. 3, pp. 910-916, 2005.

[11] H. Y. Wu, W. L. Huang, and M. H. Huang, "Direct high-yield synthesis of high aspect ratio gold nanorods," Crystal Growth \& Design, vol. 7, no. 4, pp. 831-835, 2007.

[12] C. Kan, W. Cai, C. Li, G. Fu, and L. Zhang, "Morphologic evolution and optical properties of nanostructured gold based on mesoporous silica," Journal of Applied Physics, vol. 96, no. 10, pp. 5727-5734, 2004.

[13] M. Maillard, S. Giorgio, and M. P. Pileni, "Tuning the size of silver nanodisks with similar aspect ratios: synthesis and optical properties," Journal of Physical Chemistry B, vol. 107, no. 11, pp. 2466-2470, 2003.

[14] D. A. Stuart, A. J. Haes, C. R. Yonzon, E. M. Hicks, and R. P. Van Duyne, "Biological applications of localised surface plasmonic phenomenae," IEE Proceedings Nanobiotechnology, vol. 152, no. 1, pp. 13-32, 2005.

[15] J. Pérez-Juste, I. Pastoriza-Santos, L. M. Liz-Marzán, and P. Mulvaney, "Gold nanorods: synthesis, characterization and applications," Coordination Chemistry Reviews, vol. 249, no. 17-18, pp. 1870-1901, 2005.

[16] G. Wu, A. Mikhailovsky, H. A. Khant, C. Fu, W. Chiu, and J. A. Zasadzinski, "Remotely triggered liposome release by nearinfrared light absorption via hollow gold nanoshells," Journal of the American Chemical Society, vol. 130, no. 26, pp. 8175$8177,2008$.

[17] N. P. Praetorius and T. K. Mandal, "Engineered nanoparticles in cancer therapy," Recent Patents on Drug Delivery \& Formulation, vol. 1, no. 1, pp. 37-51, 2007.

[18] P. Zijlstra, J. W. M. Chon, and M. Gu, "Five-dimensional optical recording mediated by surface plasmons in gold nanorods," Nature, vol. 459, no. 7245, pp. 410-413, 2009.

[19] C. Schönenberger, B. M. I. van der Zande, L. G. J. Fokkink et al., "Template synthesis of nanowires in porous polycarbonate membranes: electrochemistry and morphology," Journal of Physical Chemistry B, vol. 101, no. 28, pp. 5497-5505, 1997.

[20] Y. Y. Yu, S. S. Chang, C. L. Lee, and C. R. C. Wang, "Gold nanorods: electrochemical synthesis and optical properties," Journal of Physical Chemistry B, vol. 101, no. 34, pp. 66616664, 1997.

[21] F. Kim, J. H. Song, and P. Yang, "Photochemical synthesis of gold nanorods," Journal of the American Chemical Society, vol. 124 , no. 48, pp. 14316-14317, 2002.

[22] N. R. Jana, L. Gearheart, and C. J. Murphy, "Wet chemical synthesis of silver nanorods and nanowires of controllable aspect ratio," Chemical Communications, no. 7, pp. 617-618, 2001.

[23] N. Zettsu, J. M. McLellan, B. Wiley, Y. Yin, Z. Y. Li, and Y. Xia, "Synthesis, stability, and surface plasmonic properties of rhodium multipods, and their use as substrates for surfaceenhanced raman scattering," Angewandte Chemie International Edition, vol. 45, no. 8, pp. 1288-1292, 2006.

[24] Z. Li, W. Li, P. H. C. Camargo, and Y. Xia, "Facile synthesis of branched $\mathrm{Au}$ nanostructures by templating against a selfdestructive lattice of magnetic Fe nanoparticles," Angewandte Chemie International Edition, vol. 47, no. 50, pp. 9653-9656, 2008.

[25] T. K. Sau and A. L. Rogach, "Nonspherical noble metal nanoparticles: colloid-chemical synthesis and morphology control," Advanced Materials, vol. 22, no. 16, pp. 1781-1804, 2010.
[26] I. I. Naumov, Z. Li, and A. M. Bratkovsky, "Plasmonic resonances and hot spots in Ag octopods," Applied Physics Letters, vol. 96, no. 3, Article ID 033105, 2010.

[27] M. J. Mulvihill, X. Y. Ling, J. Henzie, and P. Yang, "Anisotropic etching of silver nanoparticles for plasmonic structures capable of single-particle SERS," Journal of the American Chemical Society, vol. 132, no. 1, pp. 268-274, 2010.

[28] E. Hao, G. C. Schatz, and J. T. Hupp, "Synthesis and optical properties of anisotropic metal nanoparticles," Journal of Fluorescence, vol. 14, no. 4, pp. 331-341, 2004.

[29] T. K. Sau and C. J. Murphy, "Role of ions in the colloidal synthesis of gold nanowires," Philosophical Magazine, vol. 87, no. $14-15$, pp. 2143-2158, 2007.

[30] X. Liu, R. Huang, and J. Zhu, "Functional faceted silver nanohexapods: synthesis, structure characterizations, and optical properties," Chemistry of Materials, vol. 20, no. 1, pp. 192-197, 2008.

[31] K. Park and R. A. Vaia, "Synthesis of complex Au/Ag nanorods by controlled overgrowth," Advanced Materials, vol. 20, no. 20, pp. 3882-3886, 2008.

[32] C. Li, K. L. Shuford, Q. H. Park et al., "High-yield synthesis of single-crystalline gold nano-octahedra," Angewandte Chemie International Edition, vol. 46, no. 18, pp. 3264-3268, 2007.

[33] T. K. Sau and C. J. Murphy, "Seeded high yield synthesis of short Au nanorods in aqueous solution," Langmuir, vol. 20, no. 15, pp. 6414-6420, 2004.

[34] L. Gou and C. J. Murphy, "Fine-tuning the shape of gold nanorods," Chemistry of Materials, vol. 17, no. 14, pp. 3668 3672, 2005.

[35] D. K. Smith and B. A. Korgel, "The importance of the CTAB surfactant on the colloidal seed-mediated synthesis of gold nanorods," Langmuir, vol. 24, no. 3, pp. 644-649, 2008.

[36] X. Xu and M. B. Cortie, "Shape change and color gamut in gold nanorods, dumbbells, and dog bones," Advanced Functional Materials, vol. 16, no. 16, pp. 2170-2176, 2006.

[37] K. T. Yong, Y. Sahoo, M. T. Swihart, P. M. Schneeberger, and P. N. Prasad, "Templated synthesis of gold nanorods (NRs): the effects of cosurfactants and electrolytes on the shape and optical properties," Topics in Catalysis, vol. 47, no. 1-2, pp. 4960,2008

[38] B. Nikoobakht and M. A. El-Sayed, "Preparation and growth mechanism of gold nanorods (NRs) using seed-mediated growth method," Chemistry of Materials, vol. 15, no. 10, pp. 1957-1962, 2003.

[39] C. J. Murphy, T. K. Sau, A. M. Gole et al., "Anisotropic metal nanoparticles: synthesis, assembly, and optical applications," Journal of Physical Chemistry B, vol. 109, no. 29, pp. 1385713870, 2005.

[40] Y. Xiong, H. Cai, B. J. Wiley, J. Wang, M. J. Kim, and Y. Xia, "Synthesis and mechanistic study of palladium nanobars and nanorods," Journal of the American Chemical Society, vol. 129, no. 12, pp. 3665-3675, 2007.

[41] N. R. Jana, L. Gearheart, and C. J. Murphy, "Wet chemical synthesis of high aspect ratio cylindrical gold nanorods," Journal of Physical Chemistry B, vol. 105, no. 19, pp. 40654067, 2001.

[42] N. R. Jana, L. Gearheart, and C. J. Murphy, "Seed-mediated growth approach for shape-controlled synthesis of spheroidal and rod-like gold nanoparticles using a surfactant template," Advanced Materials, vol. 13, no. 18, pp. 1389-1393, 2001.

[43] C. J. Murphy, A. M. Gole, S. E. Hunyadi, and C. J. Orendorff, "One-dimensional colloidal gold and silver nanostructures," Inorganic Chemistry, vol. 45, no. 19, pp. 7544-7554, 2006. 
[44] J. Gao, C. M. Bender, and C. J. Murphy, "Dependence of the gold nanorod aspect ratio on the nature of the directing surfactant in aqueous solution," Langmuir, vol. 19, no. 21, pp. 9065-9070, 2003.

[45] C. Kan, X. Zhu, and G. Wang, "Single-crystalline gold microplates: synthesis, characterization, and thermal stability," Journal of Physical Chemistry B, vol. 110, no. 10, pp. 46514656, 2006.

[46] C. Kan, C. Wang, J. Zhu, and H. Li, "Formation of gold and silver nanostructures within polyvinylpyrollidone (PVP) gel," Journal of Solid State Chemistry, vol. 183, no. 4, pp. 858-865, 2010.

[47] C. Kan, C. Wang, H. Li et al., "Gold microplates with welldefined shapes," Small, vol. 6, no. 16, pp. 1768-1775, 2010.

[48] Z. L. Wang, R. P. Gao, B. Nikoobakht, and M. A. El-Sayed, "Surface reconstruction of the unstable $\{110\}$ surface in gold nanorods," Journal of Physical Chemistry B, vol. 104, no. 23, pp. 5417-5420, 2000. 

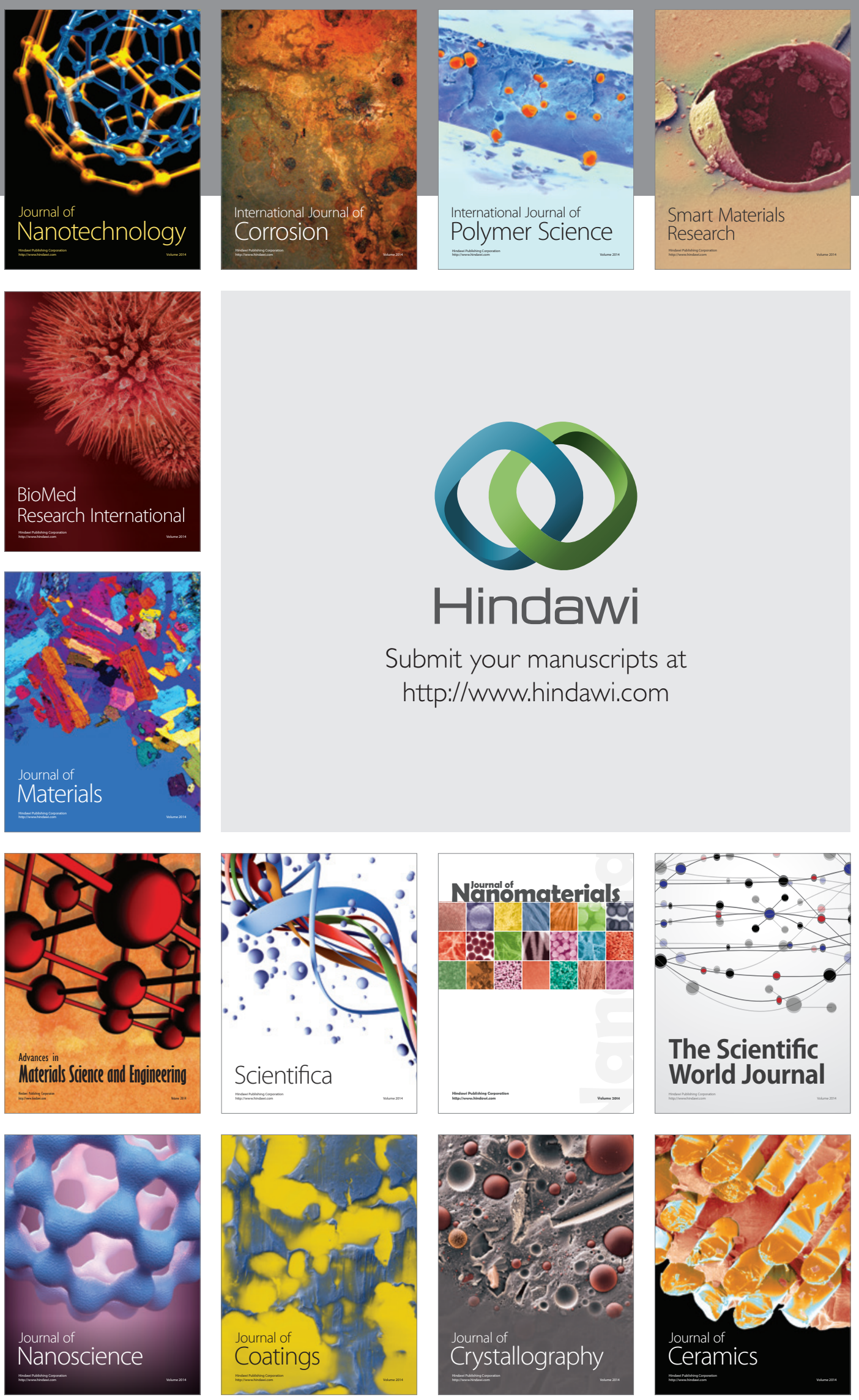

The Scientific World Journal

Submit your manuscripts at

http://www.hindawi.com

\section{World Journal}

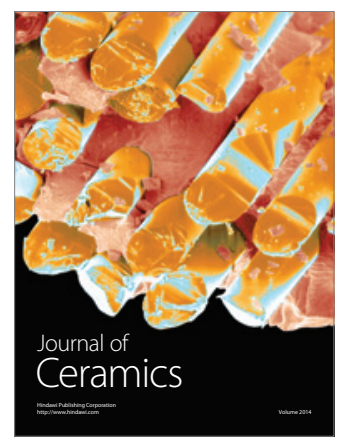

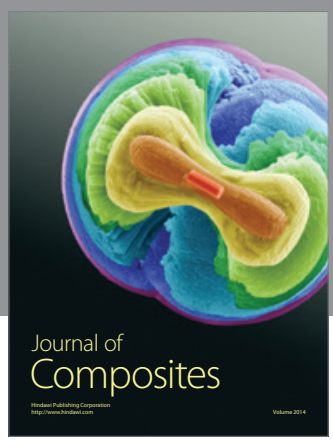
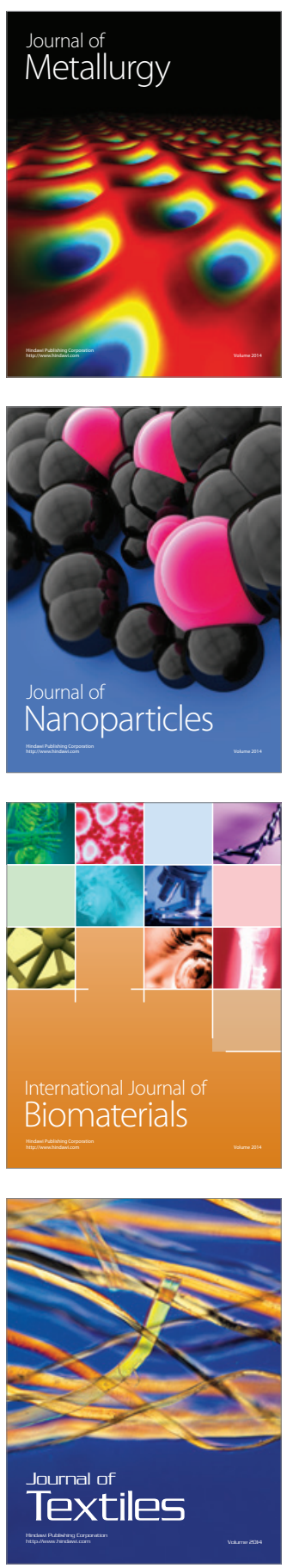